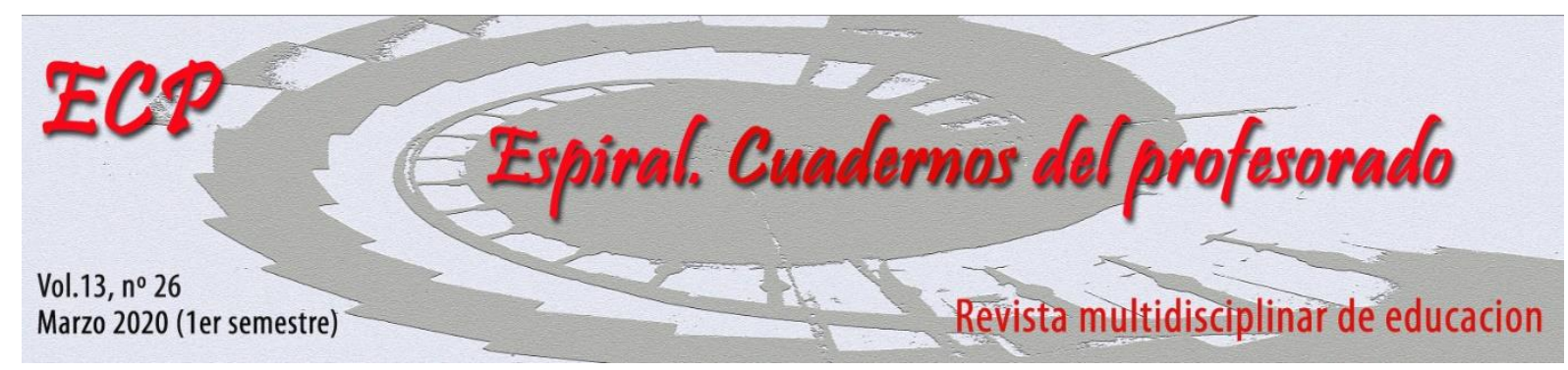

\title{
Repercusiones de un programa bioecológico en la responsabilidad y satisfacción en educación física: un estudio de caso en contextos de exclusión social
}

\section{Effects of a bioecological program in responsibility and satisfaction in physical education: a case study in contexts of social exclusion}

\author{
Javier Lamoneda Prieto \\ Consejería de Educación de la Junta de Andalucía, España.
}

\begin{abstract}
Resumen
El propósito del presente estudio fue examinar las repercusiones de un programa original de Educación Física (EF) basado en el modelo bio-ecológico sobre la responsabilidad. Además, se comprobó el grado de aceptación de la intervención. Participaron 120 estudiantes con una edad media de 14.53 años, todos pertenecientes a un centro público ubicado en una zona en riesgo de exclusión. Se empleó un estudio de caso explicativo con recogida y análisis de datos post facto. Los resultados mostraron mayor nivel de responsabilidad en EF en el grupo experimental respecto al control. Sin embargo, no se hallaron diferencias en el interés por la EF, que para ambos grupos fue satisfactoria. El contenido más valorado fueron los juegos y deportes; el objetivo de la materia más destacado, la salud; los beneficios de la asignatura, la mejora de las relaciones sociales. Se sugiere seguir avanzando en la reducción del absentismo y abandono escolar en contextos socioeconómicos desfavorecidos.
\end{abstract}

Palabras clave: compensación educativa, adolescencia, responsabilidad, educación física.

\begin{abstract}
The purpose of the present study was to examine the implications of an original Physical Education (PE) program based on the bioecological model on responsibility. In addition, the degree of acceptance of the intervention was checked. 120 students participated with an average age of 14.53 years, all belonging to a public center located in an area at risk of exclusion. An explanatory case study was used with post facto data collection and analysis. The results showed a higher level of responsibility in PE in the experimental group than in the control group. However, no differences were found in the interest in PE, which for both groups were satisfactory. The most valued content were games and sports; the objective of the most outstanding subject, health; the benefits of the subject, the improvement of social relations. It is suggested to continue making progress in reducing absenteeism and dropping out of school in disadvantaged socio-economic contexts.
\end{abstract}

Keywords: educational compensation, adolescence, responsibility, physical education.

Fecha de recepción: 17/11/2019

Fecha de aceptación: 23/01/2020

Correspondencia: Javier Lamoneda Prieto, Consejería de Educación, Junta de Andalucía, España. Email: educacionfisicajlp@gmail.com 


\section{Introducción}

Entre otras cuestiones, la educación pública tiene como reto considerar precisamente la función que esta cumple en contextos de exclusión social, en los que se ha demostrado un alto índice de absentismo (Junta de Andalucía, 2011). A este respecto, la Educación Compensatoria se caracteriza por dirigirse a aquellos sujetos que, a causa de su origen socioeconómico, padecen, o se encuentran en situación de riesgo de padecer, dificultades en su desarrollo educativo. En este contexto, la enseñanza no sólo se aborda como un ejercicio pedagógico con el fin de compensar carencias académicas o de aprendizaje, sino que contempla otros factores influyentes en la misma. Para tal fin, se requiere de un proceso sistemático y planificado desde su inicio y busca una fuerte relación entre agentes y sujetos; así mismo, de intervenciones interdisciplinares de un enfoque ecológico (Fernández, Huete, \& Vélez, 2017; Grañeras et al., 1998).

Para abordar este tipo de enseñanza, una de las corrientes dominantes ha sido el Paradigma del Déficit. Bajo este enfoque, se ha concebido el desarrollo juvenil como la ausencia de conductas problemáticas: consumo de drogas, conducta antisocial, trastornos depresivos o prácticas sexuales de riesgo (Arnett, 1999). A pesar de los logros obtenidos bajo este modelo, ha sido criticado por estancarse en patologías sin apenas considerar posibles expectativas de futuro o desarrollo de competencias (Oliva et al., 2010).

Como reacción al Paradigma de Déficit, ha surgido la denominada Positive Youth Development (Benson, Scales, Hamilton, \& Sesman, 2006). Esta corriente de pensamiento trata de superar la mera prevención o evitación (de violencia, consumo o prácticas sexuales de riesgo) a través de la promoción y desarrollo (habilidades, conductas y competencias necesarias para tener éxito en la vida social, académica y profesional como el bienestar emocional, el social y el psicológico) (Oliva et al., 2010). A pesar de que en la actualidad no existe un consenso en la definición de los elementos que constituyen el desarrollo positivo adolescente, conviene destacar dos modelos: primero, el Modelo de Desarrollo Positivo de las 5 Ces (Lerner, 2004): competencia, confianza, conexión, carácter, cuidado y compasión; y segundo, el Modelo de desarrollo positivo adolescente (Oliva et al., 2010) que consta de cinco áreas: emocional (e.g. empatía, tolerancia, optimismo), social (e.g. asertividad, resolución conflictos), cognitiva (e.g. creatividad, análisis crítico), moral (e.g. responsabilidad, prosocialidad y respeto) y de desarrollo personal (e.g. autoestima, autocontrol, iniciativa).

En relación con los proyectos que emplean la actividad física y el deporte desde una corriente positiva de desarrollo, uno de los programas con mayor difusión internacional es el denominado modelo de Responsabilidad Personal y Social (PRPS, Escartí, Buelga, Gutiérrez, \& Pascual, 2009). Los estudios originales datan de la década de los años 70 en Chicago de la mano de Donald Hellison. En síntesis, tiene como meta desarrollar las capacidades personales y sociales, la responsabilidad social tanto en el deporte como en la vida. Para ello, se propone cultivar valores de bienestar y desarrollo personal: esfuerzo y autogestión; desarrollo e integración social. El programa PRPS se une a otras investigaciones activistas que han desafiado las barreras de la marginación y exclusión social ofreciendo oportunidades de compromiso deportivo (Enright \& O'Sullivan, 2012; Oliver \& Kirk, 2014). En este sentido, los programas deportivos han demostrado ser exitosos principalmente en la mejora de las habilidades cognitivas y de la vida social y en menor medida en las habilidades de la vida emocional (Hermens, Super, Verkooijen, \& Koele, 2017).

A pesar de la creencia en los programas deportivos como un entorno en el que los jóvenes socialmente vulnerables pueden desarrollar habilidades para la vida, no existe un panorama general del desarrollo de habilidades de vida en los programas deportivos que sirven a este grupo de jóvenes (Luguetti, Oliver, Kirk, \& Dantas, 2015). Tampoco queda claro si se precisa de una metodología específica (Hellison, 2011; Theeboom, De Knop, \& Wylleman, 1993), o si se requiere de una orientación metodológica relacional más que deportiva (Haudenhuyse, Theeboom, \& Coalter, 2012).

Entre otras teorías explicativas del desarrollo humano, el modelo bioecológico ha adquirido protagonismo en su uso contemporáneo en las ciencias psicológicas y pedagógicas (Gifre \& Esteban, 2012). Según el modelo que originalmente aporta Brefenbrenner el desarrollo personal es fruto de un conjunto de procesos de interacción entre la persona y el ambiente. De tal modo que la riqueza de la

Espiral. Cuadernos del Profesorado | ISSN 1988-7701 | 2020, 13(26), 54-63 
persona es concebida por la cantidad, calidad y diversidad de interrelaciones y vínculos que se establezca.

Por todo ello, el propósito de este estudio fue examinar las repercusiones de un programa original basado en el modelo bioecológico sobre la responsabilidad personal y social en Educación Física (EF) y comprobar el grado de satisfacción de sus participantes con el programa. Se formuló como hipótesis: que los estudiantes que participaron en el programa de EF siguiendo un modelo bioecológico (grupo caso) obtendrán mejores niveles de responsabilidad personal y social que los que no siguieron ningún tratamiento (grupo control).

\section{Método}

\section{Participantes}

120 estudiantes con una edad media de 14.53 años (66 de género masculino y 54 femenino), todos pertenecientes a un centro público ubicado en una zona en riesgo de exclusión y que sigue un Programa de Compensación Educativa (Ley 17/2007, de 10 de diciembre, de Educación de Andalucía). La muestra estaba compuesta inicialmente por 154 participantes, de los cuales 34 casos fueron excluidos por no cumplimentar los instrumentos de evaluación o no obtener el consentimiento de participación en la investigación. Entre los estudiantes, 63 estaban matriculados en $1^{\circ}$ o $2^{\circ}$ de Secundaria $(46.3 \%)$ y 73 en $3^{\circ} \mathrm{o} 4^{\circ}$ de Secundaria $(53.7 \%)$, el deporte más practicado era el fútbol $(36.8 \%)$ y el modo de práctica no federado $(78.7 \%)$.

Se empleó un estudio de caso explicativo, con grupo control y experimental no equivalente para su aplicación en contextos reales y recogida y análisis de datos post facto. La asignación de cada grupo se realizó ateniendo al nivel de asistencia a clase de EF: el grupo experimental (asisten con regularidad), estaba constituido por 74 participantes (54.4\%); y el control (no asisten de manera regular y continuada), por $62(45.6 \%)$.

Para valorar el nivel de asistencia a las clases de EF se agruparon a estudiantes en función del porcentaje de faltas de asistencia injustificadas. Según la Orden de 19 de septiembre de 2005, se considera alumno/a absentista aquel que falta de manera regular y continuada sin motivo justificado; contabilizándose más de 25 horas de clase en un mes o $25 \%$ de días lectivos o de horas de clase. En este caso, se analizaron 50 sesiones de EF entre octubre de 2016 y mayo de 2017 y se contabilizaron las faltas injustificadas por alumno/a. Posteriormente se agruparon estudiantes que habían asistido a más de un $25 \%$ de las sesiones, que configuraron el grupo experimental; y por otro lado, aquellos que su porcentaje de faltas era igual o superior al $25 \%$, que formaron parte del control.

\section{Instrumentos de medida}

Responsabilidad personal y social en EF. Se utilizó la versión validada al castellano del Cuestionario de Responsabilidad personal y social en contextos de EF (Escartí et al., 2011). La escala mide dos dimensiones: 1) Responsabilidad social (ítems 1-6), e.g. ítem 2: "respeto a mi profesor"; y 2) Responsabilidad personal (ítems 7-14), e.g. ítem 11: "trato de esforzarme, aunque no me guste la tarea". Las respuestas se establecen en una escala Likert 1-5, siendo 1, "totalmente en desacuerdo", 2 "bastante en desacuerdo", 3 "poco en desacuerdo", 4 "poco de acuerdo", 5"bastante de acuerdo". El alpha de Cronbach que presentó la escala en este estudio fue de $\alpha=.81$; para la primera dimensión de $\alpha=.620 \mathrm{y}$ para la segunda de $\alpha=.785$.

Satisfacción en EF. Se recurrió al Cuestionario para el Análisis de la Satisfacción en EF (C.A.S.E.F., Moreno, Hellín, \& Hellín, 2006). Consta de 22 preguntas. A pesar de que la versión original carece de sub-división alguna, en el presente este estudio se agruparon ítems en cuatro ámbitos, atendiendo a criterios de contenido a fin de facilitar la interpretación de los resultados: (1) satisfacción con la materia de EF (ítems 5, 7, 13, 22), (2) satisfacción con los contenidos (ítems 8, 9), (3) : las características, objetivos y beneficios de la EF (ítems 14, 15, 16), (4) satisfacción con el profesor de EF (ítems 17, 18). En cualquier caso, en el tratamiento estadístico todos los ítems de la escala se valoraron de forma independiente como sugieren los autores del estudio original. 


\section{Procedimiento}

Antes de iniciar el estudio, se solicitaron permisos a los representantes legales de los estudiantes. Se informó de que sus datos serían tratados de forma anónima y que no afectarían a las calificaciones. La administración de los cuestionarios se realizó en el aula de cada grupo al iniciar la clase de EF y siempre en presencia del responsable principal de la investigación. Se recurrió al apoyo de presentaciones a fin de facilitar la comprensión de los ítems de cada cuestionario. En la explicación y cumplimentación de cuestionarios se dedicó entre 15 y 20 minutos.

Programa de intervención. Se diseñó un programa original siguiendo el modelo de desarrollo humano de Brefenbrenner. Considerando que el desarrollo es fruto de la interacción del individuo en un contexto o ambiente ecológico, se analizaron los posibles espacios de práctica de actividad física que permitiesen generar interacción y vínculos. En orden a las estructuras seriadas de Brefenbrenner (1987) los ambientes ecológicos en EF fueron un total de cinco (Figura 1): (1) Sesión de EF, (2) Actividades recreativas en tiempo de recreo; (3) Actividades deportivas en el barrio: encuentros intercentros; (4) Contacto con entidades deportivas locales: centros fitness, clubes, delegación municipal, ONGs; (5) Actividades de aventura en el medio natural en entornos lejanos.

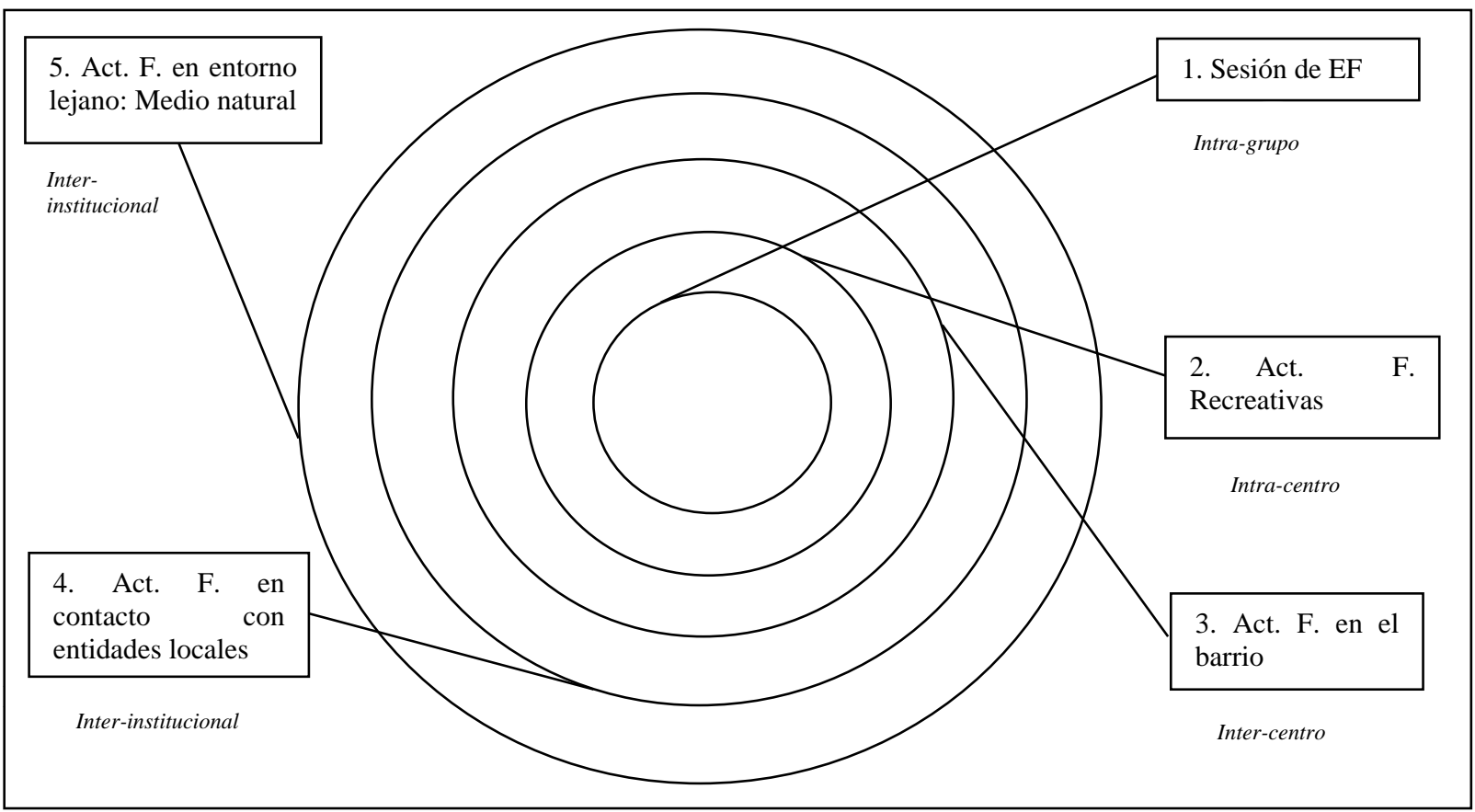

Figura 1. Ambientes ecológicos de desarrollo en EF.

La intervención se caracterizaba por llegar al aprendizaje a través de la práctica y posterior reflexión y puesta en común (Cecchini et al., 2008). Por ello, en primera instancia se empleaba la actividad física tratando de sorprender al estudiante a través del reto, el desafía, el juego y la variabilidad de práctica, que han sido mencionados medios idóneos para trabajar con jóvenes desfavorecidos (Ariza, Fernández, Soto, \& Tortosa, 2012); y al concluir, establecer tiempos de reflexión, auto-valoración y debate, por haber sido citado un recurso eficaz para trabajar la educación en valores con estos colectivos (Consejo Superior de Deportes, 2010).

\section{Análisis estadístico}

En primer lugar, se analizó la consistencia interna del cuestionario de Responsabilidad en EF mediante el alfa de Cronbach. Posteriormente, se llevaron a cabo estudios descriptivos (medias y desviaciones típicas) y análisis de la normalidad de la muestra. Los resultados de sugirieron abordar el 
estudio de las diferencias entre cada grupo (experimental-control) utilizando la prueba no paramétrica de U Mann-Whitney.

En segundo lugar, para el análisis de la satisfacción de los estudiantes en EF se analizaron frecuencias, se muestran los porcentajes de casos para cada grupo (experimental-control) y se analizan las diferencias entre grupos, empleando en este caso el test exacto de Fisher.

Todas las operaciones se realizaron a través del paquete informático para ciencias sociales IBM SPSS 20.0 para Windows con un nivel de significatividad estadística $p \leq .05$.

\section{Resultados}

\section{Responsabilidad en EF}

En la Figura 2 podemos observar diferencias significativas entre el grupo control y experimental en las variables analizadas. El grupo experimental registró mayores niveles de responsabilidad personal y social en EF (todos $p<.05$ ) que el control.

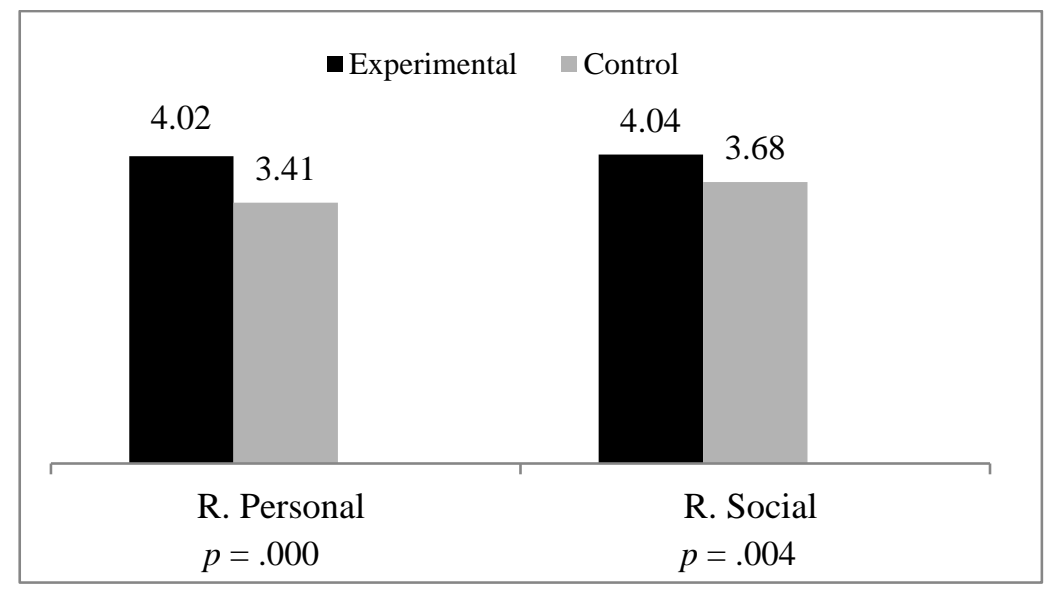

Figura 2. Media y significatividad (U Mann Whitney) para la responsabilidad en EF en el grupo experimental y control.

\section{Satisfacción general hacia la EF}

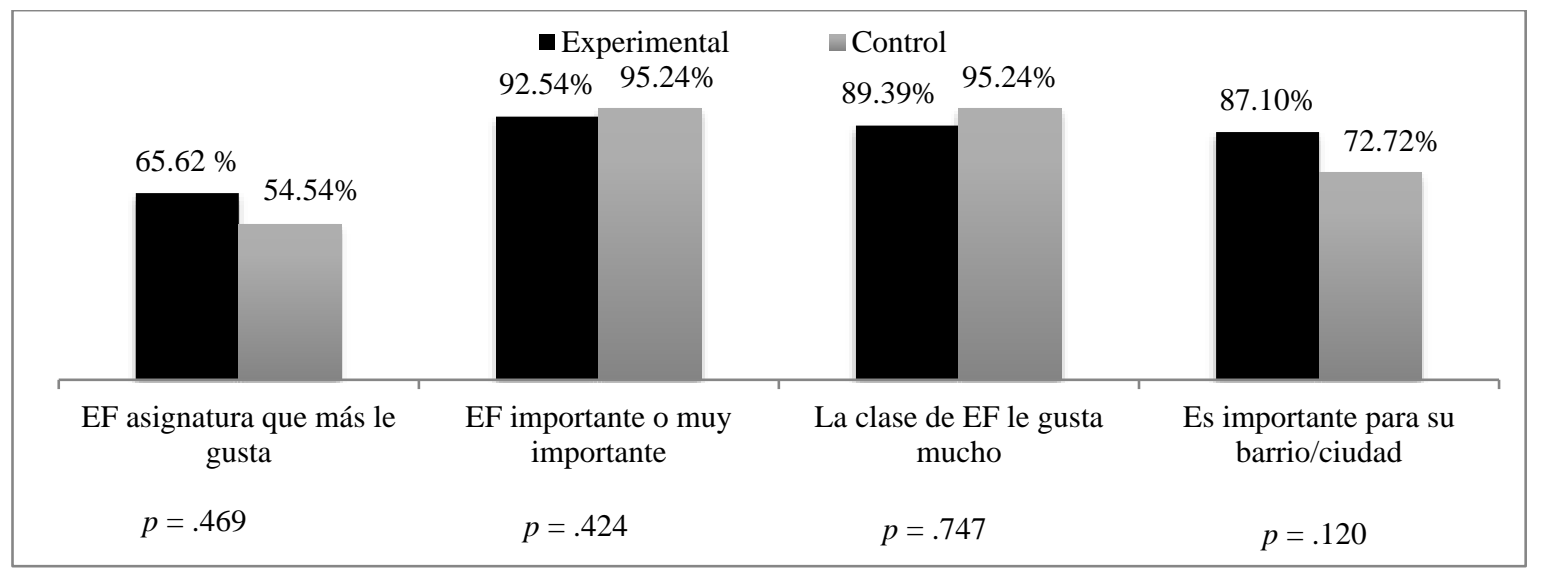

Figura 3. Frecuencias (expresadas en porcentaje) y significatividad (Fisher test) para la satisfacción general hacia la EF en el grupo experimental y control.

En la Figura 3, podemos comprobar como la falta de asistencia a la clase de EF no es una variable significativa diferencialmente con respecto a la satisfacción general de los estudiantes hacia la materia de EF, que para ambos grupos fue buena: entre un 54 y $65 \%$ de los alumnos/as señalaron que 
era la asignatura que más le gusta; más del $90 \%$ consideraron que se trata de una materia importante o muy importante; un número elevado de estudiantes (entre un 89 y $95 \%$ ) reconoció que le gustaba mucho la materia; y buena parte de los mismos (entre un 72 y 87\%) señaló que era importante para su ciudad.

\section{Valoración de con los contenidos de EF}

En la Figura 4 queda representada la valoración otorgada por los estudiantes del grupo experimental y control en relación con los bloques de contenidos de EF en Secundaria. Los resultados tanto para los contenidos mejor considerados como los menos, revelan ausencia de diferencias en los grupos estudiados (para todos $p>.05$ ). Los estudiantes coincidieron al otorgar como bloque de contenidos más valorado el de juegos y deportes (entre un 55 y 69\%) y el menos, expresión corporal (entre el 40 y $59 \%$ ).

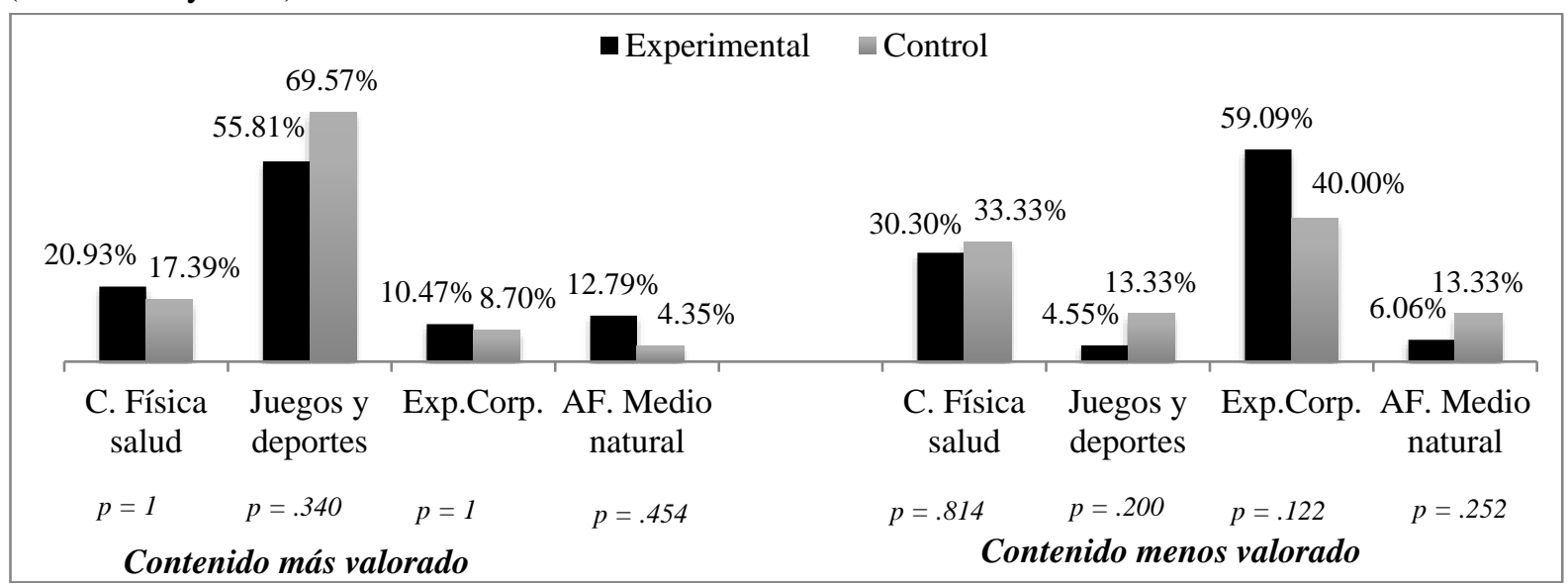

Figura 4. Frecuencias (expresadas en porcentaje) y significatividad (Fisher test) para la valoración de los contenidos de EF en el grupo experimental y control.

\section{Las características de la sesión, los objetivos y los beneficios de la EF}

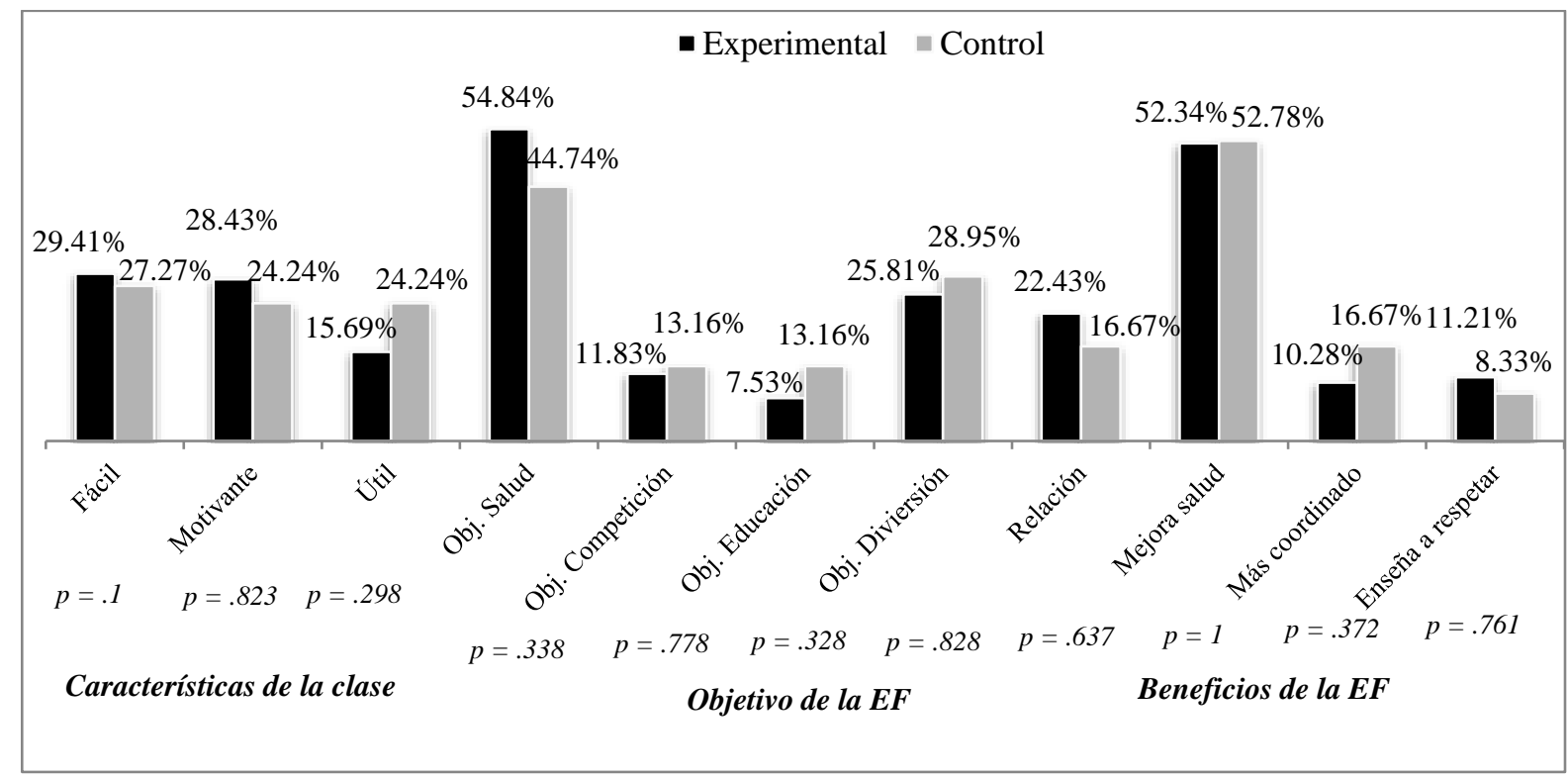

Figura 5. Frecuencias (expresadas en porcentaje) y significatividad (Fisher test) para la valoración la sesión, los objetivos y beneficios de la EF en el grupo experimental y control.

Estudiantes del grupo experimental y control coincidieron tanto la valoración de las características de la clase, los objetivos de la materia y beneficios de la EF ( $p>.05$ ) (figura 5). Para los encuestados la clase de EF se caracterizaba por ser fácil (entre el 27 y 29\%), motivante (entre el 24 y 
$28 \%$ ) y útil (entre el 15 y 24\%). Respecto a los objetivos que consideraban que debía tener la materia, destacaron principalmente dos: mejora de la salud personal (entre el 44 y 54\%) y diversión (entre el 25 y $28 \%$ ). Finalmente, en relación con los beneficios que entienden que reporta la EF coincidieron en valorar principalmente la mejora de la salud (52\%).

\section{Satisfacción con el profesor de EF}

Por lo que respecta a la consideración hacia el profesor (su actitud y características), no se apreciaron diferencias entre el grupo control como experimental $(p>.05)$ (Figura 6). Los estudiantes valoraron de la actitud del profesor de EF tener un buen trato, ser bueno con ellos (70-71\%). Y en lo que se refiere a las características del profesor, destacaron fundamentalmente: que participase activamente en clase con ellos (34-39\%) y los animase (22-31\%).

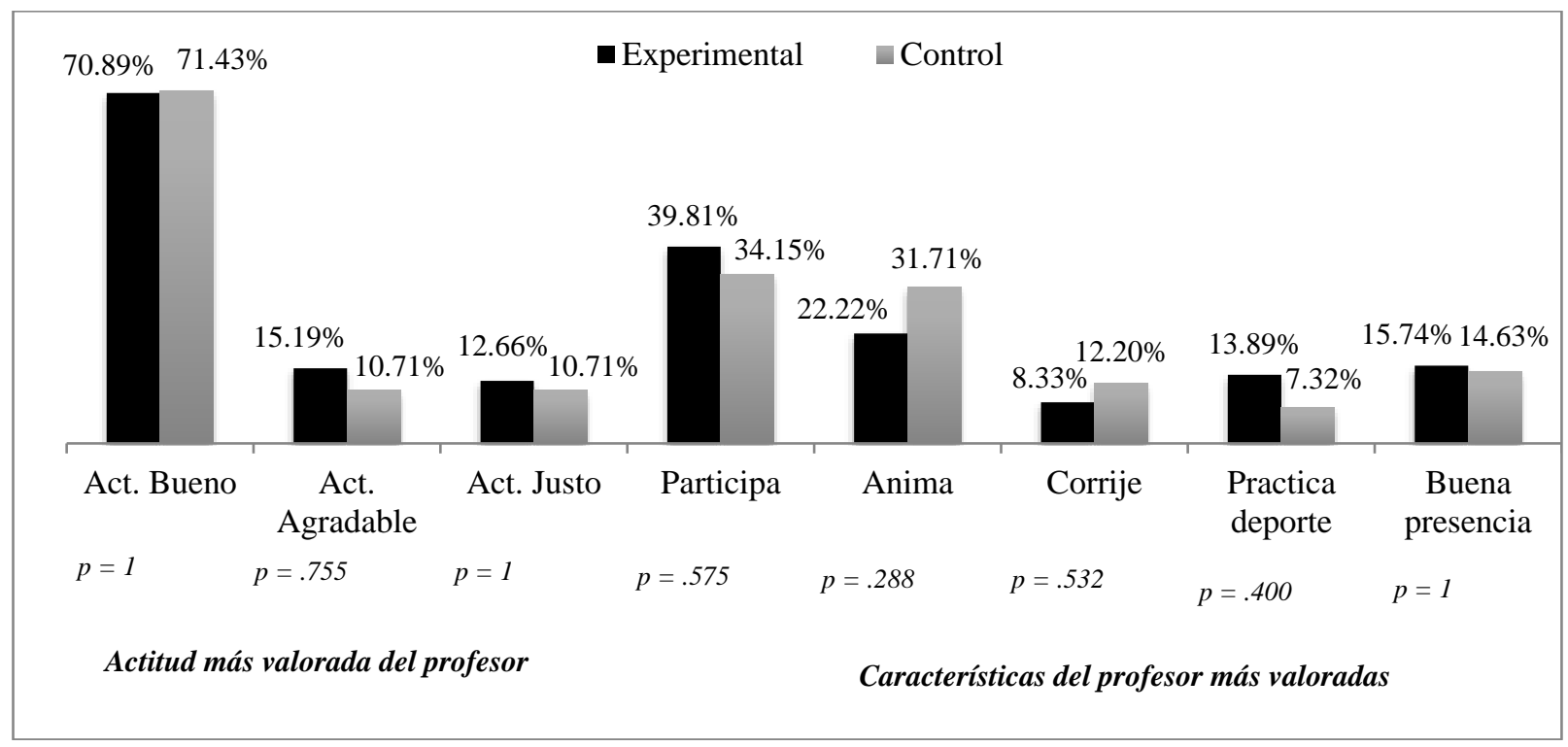

Figura 6. Frecuencias (expresadas en porcentaje) y significatividad (Fisher test) para la satisfacción con el profesor de EF en el grupo experimental y control.

\section{Discusión}

El propósito del presente estudio fue analizar cómo influye la participación en un programa bioecológico en EF en el nivel responsabilidad en EF y satisfacción hacia la EF. Los resultados mostraron diferencias entre los estudiantes del grupo experimental y control para las variables: responsabilidad en EF. En relación con el interés por la EF, que para ambos grupos no se obtuvo diferencia, los resultados fueron satisfactorios. El contenido mejor valorado fueron los juegos y deportes; la sesión de EF la consideraron fácil, motivante y útil; el objetivo de la materia más destacado, la salud; los beneficios, la mejora de las relaciones sociales; y de la intervención del profesor de EF, subrayaron que fuese bueno y participase activamente en clase.

En primer lugar, los resultados obtenidos en relación a la responsabilidad en EF permiten confirmar la hipótesis inicialmente planteada en el estudio: los participantes en un programa bioecológico en EF obtienen mayores niveles de responsabilidad personal y social en EF que los no asistentes. Estos hallazgos, pese a la limitación del estudio en el método (post facto), como el tamaño muestral, resulta interesante ser considerado debido a la necesidad que presenta la etapa adolescente de un desarrollo positivo tanto en lo personal como social (Escartí et al., 2009; Inglés et al., 2009). Estos datos se suman a los trabajos que han mostrado la actividad física y el deporte como medio a considerar en el desarrollo de jóvenes desfavorecidos. En este sentido, cabe destacar cómo la actividad deportiva es beneficiosa con grupos de exclusión social para: la mejora de habilidades emocionales de la vida, aumento de la autoestima y reducción de síntomas depresivos (Eime, Young, Harvey, Charity, \& Payne, 
2013); la mejora de habilidades cognitivas, como la fijación de metas y habilidades de autorregulación (Jonker, Elferink, \& Visscher, 2011); mejoras fisiológicas, como los cambios en los neurotransmisores asociados con el mejoramiento del bienestar (Lubans, Plotnikoff, \& Lubans, 2012; y la mejora de las habilidades sociales, como la comunicación, la resolución de conflictos y la empatía (Bailey, Hillman, Arent, \& Petitpas, 2013).

En lo que respecta a la valoración de los estudiantes de la materia de EF, en todos los casos el contenido más valorado fueron los juegos y deportes. Respecto a la sesión de EF, la describieron: fácil, motivante y útil; el objetivo de la materia más destacado, la salud; y los beneficios, la mejora de las relaciones sociales. Coincidimos en este caso con anteriores estudios que emplean la actividad deportiva con una orientación recreativa y relacional en jóvenes en riesgo de exclusión social (Rodríguez, Esquivel, Rodríguez, \& Fonseca, 2016).

En relación al tipo de intervención docente, independientemente del grupo al que pertenecían destacaron que fuese "bueno" con ellos y participase activamente. Aunque resulta atrevido desenmascarar el modelo de profesor eficaz en contextos desfavorecidos, conviene tener en consideración los hallazgos obtenidos y profundizar en el análisis en futuros estudios. En este caso, coincidimos con los trabajos que enfatizan la figura del profesor / entrenador como generador de relaciones positivas con los jóvenes (Hermens et al., 2017). En esta misma línea argumental, Luguetti et al. (2015 y 2019) recalcaron la importancia de emplear en los programas deportivos con jóvenes de origen vulnerable un enfoque activista y una pedagogía centrada en el estudiante a través de la participación activa y colaborativa. En este sentido la propuesta alude a una situación en la sesión interna y participativa al haberse demostrado que facilita el buen seguimiento de los estudiantes y promueve un alto nivel de afectividad (Pacheco \& Chacón en Sánchez, 2002). Desde similar enfoque, en la pedagogía de los oprimidos Freire (1987) aborda el proceso educativo desde el la pedagogía amor que lleva al cuidado y el compromiso con los jóvenes.

Los resultados que arroja este trabajo convienen ser considerados con cautela debido a las limitaciones con las que cuenta el tipo de diseño empleado. En primer lugar, la valoración post facto no ha permitido analizar la evolución de los datos en cada grupo. En segundo lugar, en un estudio como el que se presenta, con grupos vulnerables, es altamente de interés controlar un mayor número de variables que influyen en el estudiante como son físicos o extraescolares, familiares y el resto de prácticas pedagógicas fuera del programa de $\mathrm{EF}$.

A pesar de las limitaciones expuestas, el modelo de bioecológico ha sido desarrollado con éxito en el ámbito educativo dirigido a niños/as desfavorecidos (Besalú \& Vila, 2007; Gifre \& Esteban, 2012). Este tipo de intervención comunitaria ha sido objeto de políticas sociales como Segundo Tempo (Rossi, de Alencar, Rossi, \& Pereira, 2014) en Brasil, mediante la apertura de instalaciones deportivas a escuelas públicas y áreas periféricas. En esta misma línea el gobierno inglés ha financiado clubes deportivos que promoviesen la actividad física, especialmente en colectivos con dificultad de acceso (NAO, 2012).

\section{Conclusiones}

La configuración de ambientes ecológicos a través de la EF en los programas de compensación educativa puede resultar interesantes en el desarrollo positivo del estudiante, no solo por las diferencias en los niveles de responsabilidad personal y social entre estudiantes que participaron y absentistas, sino también, porque para todos resultó ser un programa altamente gratificante.

La asistencia regular y continuada a programas de compensación educativa en EF resultan ser valiosas en el desarrollo positivo del estudiante. Por esta razón, es crucial seguir avanzando en la reducción del absentismo y abandono escolar en contextos socioeconómicos desfavorecidos.

Por otra parte, conviene considerar la materia de EF como medio idóneo para el trabajo con jóvenes desfavorecidos, ya que ha sido demostrado como independientemente del nivel de asistencia de estos todos muestran alto interés por la materia. 
En relación con el modo de afrontar la intervención docente en EF compensatoria, cabe destacar el alto valor atribuido a la figura del profesor: que tenga buen trato con los estudiantes, adquiera un alto nivel de compromiso, sea participativo y genere en el grupo relaciones sociales favorables.

Finalmente, puede resultar interesante tener en consideración la concepción de EF de este tipo de estudiantes a fin de abordar futuras intervenciones educativas con éxito: que promueva hábitos de vida saludables y relaciones sociales más estrechas a través de sesiones lúdicas, motivantes, sencillas y al mismo tiempo útiles.

\section{Referencias}

Álvarez, D., Rodríguez, C., González, P., Núñez, J. C., \& Álvarez, L. (2010). La formación de los futuros docentes frente a la violencia escolar. Revista de Psicodidáctica, 15(1), 35-56.

Ariza, M. C., J, Fernández, Mª. Ta., Soto, F. J., \& Tortosa F. (Coords.) (2012). Respuestas flexibles en contextos educativos diversos. Murcia: Consejería de Educación, Formación y Empleo.

Arnett, J. (1999). Adolescent Storm and Stress, Reconsidered. American Psychologist, 54, 317-326.

Bailey, R., Hillman, C., Arent, S., \& Petitpas, A. (2013). Physical activity: An underestimated investment in human capital? Journal of Physical Activity and Health, 10, 289-308. doi:10.1123/jpah.10.3.289

Besalú, X. \& Vila, I. (2007). La buena educación. Madrid: Los libros de la Catarata.

Benson, P. L., Scales, P. C., Hamilton, S. F., \& Sesman, A. J. (2006). Positive youth development: Theory, research and applications. En R.M. Lerner (Ed.), Theoretical models of human development. Volume 1 of Handbook of Child Psychology, (6 ${ }^{\mathrm{a}}$ ed.), (pp.894-941). Editors-in-chief: W. Damon y R. M. Lerner. Hoboken, NJ: Wiley.

Bronfenbrenner, U. (1987). La ecología del desarrollo humano. Experimentos en entornos naturales y diseñados. Barcelona: Paidós.

Cecchini, J. A., Fernández, J., González, C., \& Arruza, J.A. (2008). Repercusiones del Programa Delfos de educación en valores a través del deporte en jóvenes escolares. Revista de Educación, 346, 167-186.

Consejo Superior de Deportes (2010). Deporte actividad física e inclusión social. Una guía para la intervención social a través de las actividades deportivas. Plan A+D. Programa: Actividad física, deporte e inclusión social. Madrid: CSD.

Eime, R. M., Young, J. A., Harvey, J. T., Charity, M. J., \& Payne, W. R. (2013). A systematic review of the psychological and social benefits of participation in sport for children and adolescents: Informing development of a conceptual model of health through sport. International Journal of Behavioral Nutrition and Physical Activity, 10, 98. doi:10.1186/1479-5868-10-98

Enright, E. \& O'Sullivan, M. (2012). Producing different knowledge and producing knowledge differently: Rethinking physical education research and practice through participatory visual methods. Sport, Education and Society, 17(1), 35-55. doi:10.1080/13573322.2011. 607911

Escartí, A., Buelga, S., Gutiérrez, M., \& Pascual, C. (2009). El desarrollo positivo a través de la actividad física y el deporte: el programa de responsabilidad personal y social. Revista de Psicología del Deporte, 62(12), 45-52.

Escartí, A., Gutiérrez, M., \& Pascual, C. (2011). Propiedades psicométricas de la versión española del cuestionario de responsabilidad personal y social en contextos de educación física. Revista de Psicología del Deporte, 20(1), 119-130.

Fernández, J., Huete, A., \& Vélez, L. (2017). Vulnerable groups at risk for sport and social exclusion. Journal of Physical Education and Sport, 17(1), 312-326. doi:10.7752/jpes.2017.01047

Freire, P. (1987). Pedagogia do Oprimido [Pedagogy of the oppressed] (17 th ed.). Río de Janeiro: Paz e Terra.

Gifre, M. \& Esteban, M. (2012). Consideraciones educativas de la perspectiva ecológica de Urie Bronfenbrenner. Contextos Educativos, 15, 79-92.

Grañeras, M., Lamelas, R., Segalerva, A., Vázquez, E., Gordo, J. L., \& Molinevo, J. (1998). Catorce años de investigación sobre las desigualdades en educación en España. Centro de investigación y documentación educativa. Área de Estudios e Investigación Ministerio de Educación y Cultura. 
Haudenhuyse, R.P., Theeboom, M., \& Coalter, F. (2012). The potential of sports-based social interventions for vulnerable youth: implications for sport coaches and youth workers. Journal of Youth Studies, 15(4), 437-454. doi:10.1080/13676261.2012.663895

Hellison, D. (2011). Teaching responsibility through physical activity (3er ed.). Champaign, IL: Human Kinetics.

Hermens, N., Super, S., Verkooijen, K. T., \& Koele, M.A. (2017). A Systematic Review of Life Skill Development Through Sports Programs Serving Socially Vulnerable Youth. Research quarterly for exercise and sport doi:10.1080 / 02701367.2017 .135552

Inglés, C. J., Benavides, G., Redondo, J., García, J. M., Ruiz, C., Estévez, C., \& Huéscar, E. (2009). Conducta prosocial y rendimiento académico en estudiantes españoles de Educación Secundaria Obligatoria. Anales de Psicología, 25(1), 93-101.

Jonker, L., Elferink, M. T., \& Visscher, C. (2011). The role of self-regulatory skills in sport and academic performances of elite youth athletes. Talent Development and Excellence, 3, 263-275.

Junta de Andalucía (2007). Ley 17/2007, de 10 de diciembre, de Educación de Andalucía (BOJA n 252, de 26 de diciembre).

Junta de Andalucía (2005). Orden de 19 de septiembre de 2005, por la que se desarrollan determinados aspectos del Plan Integral para la Prevención, Seguimiento y Control del absentismo Escolar (BOJA no 202 de 17 de octubre).

Junta de Andalucía (2011). Acuerdo de 30 de diciembre de 2011, del Consejo de Gobierno, por el que se aprueba el Plan Educativo de Zona para el Polígono Sur de Sevilla (BOJA nº 9, de 16 de enero).

Lerner, R.M. (2004). Liberty: Thriving and civic engagement among American youth. Thousand Oaks. CA: Sage.

Lubans, D. R., Plotnikoff, R. C., \& Lubans, N. J. (2012). Review: A systematic review of the impact of physical activity programs on social and emotional well-being in at-risk youth. Child and Adolescent Mental Health, 17, 2-13. doi:10.1111/j.1475-3588.2011.00623.x

Luguetti, C., Kirk, D., \& Oliver, K. L (2019). Towards a pedagogy of love: exploring pre-service teachers' and youth's experiences of an activist sport pedagogical model, Physical Education and Sport Pedagogy, 24(6), 629-646. doi:10.1080/17408989.2019.1663499

Luguetti, C., Oliver, K. L., Kirk, D., \& Dantas, L. (2015). Exploring an activist approach of working with boys from socially vulnerable backgrounds in a sport context. Sport, Education and Society, 1-18. Doi: 10.1080/13573322.2015.1054274

Moreno, J. A., Hellín, P., \& Hellín, G. (2006). Pensamiento del alumno sobre la educación física según la edad. Apunts: Educación Física y Deportes, 85, 28-35

NAO (2012). An update on the government's approach to tackling obesity. London: National Audit Office.

Oliva, A., Ríos, M., Antolín, L., Parra, A., Hernando, A., \& Pertegal, M. A. (2010). Más allá del déficit: construyendo un modelo de desarrollo positivo adolescente. Infancia y Aprendizaje, 33(2), 223-234.

Oliver, K. L. \& Kirk, D. (2014). Towards an activist approach to research and advocacy for girls and physical education. Physical Education \& Sport Pedagogy, 1-15. Doi:10.1080/17408989.2014. 895803

Rodríguez, A., Esquivel, M. J., Rodríguez, H., \& Fonseca, H. (2016). Effect of a program of sport-recreational activities on aggression and prosocial values among youth living in social risk. European Journal of Human Movement, 37, 143-162.

Rossi, R., de Alencar, R., Rossi, M., \& Pereira, A. (2014). Sport and social inclusion. Study of the program "segundo tempo" in Feira de Santana, Bahia - Brazil. Journal of Physical Education and Sport, 14(4), 446-454.

Sánchez, F. (2002). Didáctica de la Educación Física. Madrid: Prentice Hall.

Theeboom, M., De Knop, P., \& Wylleman, P. (1993). Underprivileged youth: a forgotten target group within a sports policy? In: W. Duquet, P. De Knop \& L. Bollaert, eds. Youth sport: a social approach. Brussel: VUBPRESS. 